\title{
Peripheral myopization and visual performance with experimental rigid gas permeable and soft contact lens design
}

\author{
J. Pauné ${ }^{a}$, A. Queiros ${ }^{b}$, L. Quevedo $^{c}$, H. Neves $^{b}$, D. Lopes-Ferreira ${ }^{b}$, \\ J.M. González-Méijome ${ }^{\mathrm{b}, *}$ \\ a Centro Medico Teknon, Barcelona, Spain \\ b Clinical \& Experimental Optometry Research Lab (CEORLab), Center of Physics, University of Minho, Braga, Portugal \\ c Universidad Politécnica de Cataluña, Terrasa, Spain
}

\section{A R T I C L E I N F O}

\section{Article history:}

Received 25 April 2013

Received in revised form 10 August 2014

Accepted 20 August 2014

\section{Keywords:}

Myopia

Custom-design

Contact lenses

Peripheral refraction

\begin{abstract}
A B S T R A C T
Purpose: To evaluate the performance of two experimental contact lenses $(\mathrm{CL})$ designed to induce relative peripheral myopic defocus in myopic eyes.

Methods: Ten right eyes of 10 subjects were fitted with three different CL: a soft experimental lens (Exp$\mathrm{SCL}$ ), a rigid gas permeable experimental lens (ExpRGP) and a standard RGP lens made of the same material (StdRGP). Central and peripheral refraction was measured using a Grand Seiko open-field autorefractometer across the central $60^{\circ}$ of the horizontal visual field. Ocular aberrations were measured with a Hartman-Shack aberrometer, and monocular contrast sensitivity function (CSF) was measured with a VCTS6500 without and with the three contact lenses.

Results: Both experimental lenses were able to increase significantly the relative peripheral myopic defocus up to $-0.50 \mathrm{D}$ in the nasal field and $-1.00 \mathrm{D}$ in the temporal field $(p<0.05)$. The ExpRGP induced a significantly higher myopic defocus in the temporal field compared to the ExpSCL. ExpSCL induced significantly lower levels of Spherical-like HOA than ExpRGP for the $5 \mathrm{~mm}$ pupil size $(p<0.05)$. Both experimental lenses kept CSF within normal limits without any statistically significant change from baseline $(p>0.05)$.

Conclusions: RGP lens design seems to be more effective to induce a significant myopic change in the relative peripheral refractive error. Both lenses preserve a good visual performance. The worsened optical quality observed in ExpRGP was due to an increased coma-like and spherical-like HOA. However, no impact on the visual quality as measured by CSF was observed.
\end{abstract}

(C) 2014 British Contact Lens Association. Published by Elsevier Ltd. All rights reserved.

\section{Introduction}

The potential relevance of relative peripheral optical defocus and image quality on the emmetropization mechanism has been a matter of interest among scientists. Evidence suggests that hyperopic defocus in the peripheral retina is associated with myopic progression in animals [1]. In human eyes, several studies have shown that in myopes, the relative peripheral refraction tended to be hyperopic [2-4] and helped to explain the progression in myopia. Furthermore, East Asian myopes showed higher values of peripheral hyperopia compared to white myopes of the same degree [5]. Orthokeratology has been shown to cause significant

\footnotetext{
* Corresponding author at: Clinical \& Experimental Optometry Research Lab, Department of Physics (Optometry), University of Minho, 4710-057 Braga, Portugal. Tel.: +35125360 4320; fax: +351253678981.

E-mail address: jgmeijome@fisica.uminho.pt (J.M. González-Méijome).
}

changes in peripheral optics. These changes were found to be significantly different from those found in conventional treatments such as spectacles [6,7] or single vision soft CL [8]. Although the biological mechanism behind this slowing process is not fully understood, it has been postulated to be related to the myopic relative peripheral refraction induced by orthokeratology treatment $[9,10]$.

It is therefore the manipulation of peripheral defocus that has been proposed as a possible mechanism to slow-down myopia development and this can be achieved with ophthalmic lenses [11] or CL $[12,13]$.

CLs move with the eye thus can align with the optical system of the eye. They can be custom-designed to correct the central visual field and to create a desired optical effect in the peripheral field [14]. Some commercially available CL were found to create similar effect as that created by orthokeratology treatment in some reports $[13,15,16]$. The effectiveness in retarding myopic progression by these lens designs has been evaluated in some clinical studies [13]. Most of the available lenses for myopia retention are now 
manufactured in soft materials $[13,17]$. However, the potential of rigid gas permeable (RGP) lens materials should not be neglected as their surfaces are not easily distorted by the underlying corneal topography. Thus, RGP materials might be more effective in reproducing a desired optical effect and might provide a benefit for the future development of optical solutions to modify the refraction pattern across the peripheral retina.

The purpose of this work was to evaluate the impact of customdesigned soft and rigid gas permeable CL to create a peripheral myopic defocus in the peripheral field and to evaluate the optical and visual performance. To our knowledge, no previous study has evaluated the impact of peripheral gradient lenses for myopia regulation on optical quality and visual performance using wavefront technology and contrast sensitivity function, respectively.

\section{Methods}

\subsection{Sample}

The right eyes of 10 myopic female subjects aged 21-26 years $(23.40 \pm 1.84$ years $)$ with spherical equivalent refraction ranging from $-1.25 \mathrm{D}$ to $-6.50 \mathrm{D}(-3.37 \pm 1.28 \mathrm{D})$ and refractive astigmatism below $-1.00 \mathrm{D}$ were enrolled. This was a non-dispensing study and subjects were enrolled from the research staff working in the Clinical and Experimental Optometry Research Lab (CEORLab, University of Minho, Braga, Portugal). They received information about the purpose and study procedures and gave written informed consent in accordance with the tenets of the Declaration of Helsinki. Inclusion criteria required that the subjects were free from any ocular disease and were not taking any systemic or ocular medication which would affect the refractive error or the accommodative function, and their best corrected visual acuity should be $20 / 20$ or higher with the best spectacle correction.

\subsection{Lenses}

A standard RGP lens (StdRGP), an experimental RGP (ExpRGP) lens and an experimental soft lens (ExpSCL) designed to produce peripheral myopic defocus were fitted on the right eyes of the recruited subjects. Randomization was done to choose which lens was first, second and third to be worn after a baseline measurement had been obtained on the naked eye. Technical details of the lenses used are presented in Table 1. Optical design of the experimental lenses was conducted using parameters for theoretical eye obtained from Atchison [18] and Zemax-EE software v.6 (Radiant ZEMAX, WA, USA). Both experimental lenses have a unique central back and front aspheric optic zone of $9 \mathrm{~mm}$ of diameter. These lenses do not have a central zone of single vision. Instead, only the central apical zone has the distance power required for distance vision and the aspheric design provides a progressive increasing add power, starting from the central geometrical point. This design affords $+1.50 \mathrm{D}$ add increase at $2 \mathrm{~mm}$ from center $(4 \mathrm{~mm}$ chord diameter) corresponding to approximately $30^{\circ}$ of retinal eccentricity, and achieving around $+6.5 \mathrm{D}$ at the edge of the optic zone $(9 \mathrm{~mm}$ chord diameter). Both ExpSCL and ExpRGP were designed to have the same power change from center to periphery and both had the same optic zone diameter.

Lenses were fitted according the topographical information ( $\operatorname{simK}$ readings measured over the $3 \mathrm{~mm}$ of the central cornea and eccentricity over a chord diameter of $9.0 \mathrm{~mm}$ ). Trial lenses were used to achieve optimal fitting in a pre-study visit. Lenses were ordered based on the vertexed spherical equivalent refraction. Over-refraction was done at the trial visit over the contact lenses and a new lens was ordered if discrepancies over \pm 0.25 were found. Fitting was assessed for centration and lag on lateral gaze movements using a graticule eyepiece in the slit-lamp. All lenses were within the desired limits of less than $0.5 \mathrm{~mm}$ of decentration on primary gaze direction and less than $0.5 \mathrm{~mm}$ of lag on lateral gaze direction. This criteria was applied to the SCL and RGP lenses. Large diameter and fitting approach motivated that the RGP lenses had a limited decentration and lag on lateral gaze and blinking. These values are reported as acceptable good fitting parameters for modern soft contact lenses [19]. RGP lenses use to decenter and move more. However the large diameter used in these lenses allow to meet similar dynamic behavior and the same criteria was applied. In the context of treatments to reduce myopia progression, centration might be of paramount relevance to induce the desired effect in both the nasal and temporal field [20]. On the study visit, lenses were allowed to settle for $20-30 \mathrm{~min}$ to equilibrate and stabilize at the ocular surface, and for the subject to feel comfortable enough to conduct the examination without excessive tearing or reflex blinking. This period was enough to eliminate excessive tearing and reflex blinking with the RGP lenses in all subjects. Baseline (naked eye) measurements were obtained without correction for peripheral refraction and aberrations and with the best spectacle correction in a trial frame at $11 \mathrm{~mm}$ for CSF.

\subsection{Peripheral refraction}

The measurement of central and peripheral (off-axis) refraction were obtained with an open-field Grand Seiko AutoRefractometer/Keratometer WAM-5500 (Grand Seiko Co., Ltd., Hiroshima, Japan) up to $30^{\circ}$ in the nasal and temporal horizontal field in $5^{\circ}$ steps. This instrument and its other commercial brand using the same technology for refractive error measurement (ShinNippon) have been reliably used in foveal $[21,22]$ and peripheral refraction measurements [10,23]. For the purpose of this study, a laser system was mounted on top of the subjects head and aligned with the central fixation point in primary gaze. Peripheral refraction were measured with head rotation to ensure that the lens did not displace from the resting position in primary gaze. The large diameter of the lens and the fitting approach ensured that the lens did not experience random horizontal or vertical displacement. To measure the rotation of the head, the laser had to coincide with a series of markings in the wall in front of the subject at a distance of $2.5 \mathrm{~m}$. This created a limitation on the range of field measured, whereas in our previous studies we were able to measure up to $40^{\circ}$ to each side of the visual field by rotating the eye $[10,15,23]$. The left eye was occluded during the measurements to avoid misalignments under binocular fixation. Measurements were conducted under non-cycloplegic conditions.

Descriptive statistics (mean \pm SD) were calculated for the refraction vector components (M, J0 and J45) of refractive errors, as recommended by Thibos [24]. Peripheral measurements were done by using the pupil center for alignment. M, J0 and J45 considered for statistical analysis resulted from the average of the 5 consecutive readings obtained at each visual field eccentricity. Relative peripheral refractive error (RPRE) was calculated by subtracting the central M, J0 or J45 value obtained at the fovea from that obtained at each eccentric retinal location.

\subsection{Optical quality}

Optical quality of the eye was assessed using an Irx3 HartmanShack aberrometer (ImagineEyes, Orsay, France). Higher order aberrations (HOA) from 3rd to 6th order were obtained for a 3 and $5 \mathrm{~mm}$ pupil size. Three repeated measurements were obtained under each experimental condition and then averaged for statistical analysis. Under the room illumination used, all wavefront measures warranted a minimum of $5 \mathrm{~mm}$ round pupil size without pharmacological dilation in all cases. Changes in root mean square 
Table 1

Technical details of the lenses used in the study.

\begin{tabular}{|c|c|c|c|}
\hline Material (USAN) & $\begin{array}{l}\text { StdRGP } \\
\text { Boston XO2 (hexafocon B) }\end{array}$ & $\begin{array}{l}\text { ExpRGP } \\
\text { Boston XO2 (hexafocon B) }\end{array}$ & $\begin{array}{l}\text { ExpSCL } \\
\text { Benz G3X (hioxifilcon B) 49\% EWC }\end{array}$ \\
\hline Oxygen permeability (barrer) & 141 & 141 & 15 \\
\hline Refractive index & 1.424 & 1.424 & 1.425 \\
\hline Overall diameter (mm) & 10.6 & 10.5 & 14 \\
\hline Surface geometry anterior/posterior & Aspheric/aspheric & Aspheric/aspheric & Aspheric/spherical \\
\hline Optic zone diameter & 9.0 & 9.0 & 9.0 \\
\hline Base curve radius (mm) & $7.50-8.20$ & & 8.6 \\
\hline
\end{tabular}

Standard RGP lens (StdRGP); Experimental RGP (ExpRGP); Experimental soft lens (ExpSCL); EWC: equilibrium water content.

(RMS) from baseline (no lens wear) for spherical-like HOA (including Zernike polynomials $Z_{4}{ }^{0}$ and $Z_{6}{ }^{0}$ ), coma-like HOA (including Zernike polynomials $Z_{3}{ }^{-1}, Z_{3}{ }^{1}, Z_{5}{ }^{-1}$ and $Z_{5}{ }^{1}$ ), secondary astigmatism HOA (including Zernike polynomials $Z_{4}{ }^{-2}, Z_{4}{ }^{2}, Z_{6}{ }^{-2}$ and $Z_{6}{ }^{2}$ ) and Total HOA were considered for statistical analysis.

\subsection{CSF measurements}

Visual performance was assessed using the monocular Contrast Sensitivity Function (CSF) with the Vision Contrast Test System VCTS6500 (Vistech, Dayton, OH) for spatial frequencies of 1.5, 3, 6,12 and 18 cycles/degrees.

\subsection{Statistical analysis}

The SPSS software package v.19 (SPSS Inc., Chicago, IL, USA) was used for statistical analysis. Given the small sample size, nonparametric analyses were conducted using Wilcoxon Signed Ranks Test to evaluate differences between the results of each CL compared to baseline values for the same variables. Kruskal-Wallis with post hoc correction for multiple comparisons was used to compare the results of relative peripheral refractive error induced by the three lenses. Correlations between peripheral refraction, HOA and contrast sensitivity have been evaluated using Spearman Rho correlation coefficient. For statistical purposes, a $p$ value lower than 0.05 was considered statistically significant. Bonferroni post hoc correction was applied when multiple comparisons were involved among the three experimental conditions and the no-lens condition. All $p$ values reported include the correction for multiple comparisons.

\section{Results}

Fig. 1A-C show the changes induced by each contact lens in the RPRE compared to baseline values (without contact lenses) in terms of M, J0 and J45, respectively. Differences were statistically significant for 25 and $30^{\circ}$ of temporal eccentricity between Baseline and both experimental lenses as well as between ExpRGP vs ExpSCL and ExpRGP vs StdRGP. Differences between ExpRGP vs baseline and ExpRGP vs StdRGP in the nasal field were statistically significant $(p<0.013$, Wilcoxon signed ranks test). Differences in J0 astigmatic component were statistically significant between baseline and both experimental lenses $(p<0.001$ and $p=0.010$ for ExpRGP and StdRGP, respectively) as well as between ExpRGP vs StdRGP for $30^{\circ}$ in the temporal field $(p=0.007)$. Furthermore, there were significant differences at 25 and $30^{\circ}$ for ExpRGP vs baseline $(p=0.010)$ and from 10 to $30^{\circ}$ for ExpRGP vs $\operatorname{StdRGP}(p<0.007)$ in the temporal field. None of the comparisons for J45 component showed statistically significant differences between conditions under evaluation ( $p>0.05$ in all comparisons).

HOA data for 3- and 5-mm pupil diameter are presented in Fig. 2A and B, respectively. Optical quality was worsened by the ExpRGP lens compared to baseline and StdRGP for sphericallike HOA, coma-like HOA and total HOA $(p<0.05)$; furthermore, ExpRGP also showed significantly higher Total HOA compared to StdRGP $(p=0.015)$. All these statistically significant differences were noticed for 5-mm pupil, except for ExpRGP vs baseline at $3-\mathrm{mm}$ pupil size $(p=0.033)$. As expected, HOA were significantly higher for 5-mm pupil size compared to $3-\mathrm{mm}$ pupil size $(p<0.004$ for all orders). StdRGP lens did not induce any significant change in HOA compared to baseline $(p>0.05)$.

Monocular CSF remained within normal limits provided by the manufacturer for the test, for all conditions evaluated. Furthermore, there were no statistically significant differences between spectacle-corrected CSF at baseline and StdRGP, ExpRGP or ExpSCL $(p>0.05$, Wilcoxon signed ranks test) or between StdRGP and ExpSCL for all the range of frequencies evaluated.

Correlations between relative peripheral refractive error (RPRE) and HOA were statistically significant for both experimental lenses. Spherical-like HOA (RMS resulting from 4th to 6th-order SA) for a $5 \mathrm{~mm}$ pupil presented a negative correlation with RPRE such that RPRE become more negative as SA RMS became more positive (Pearson Rho $\leq-0.635, p \leq 0.022$ ). Coma-like aberrations also presented a negative and significant correlation for the ExpRGP lens (Pearson Rho $=-0.638 ; p=0.047$ ) but not for the ExpSCL or StdRGP lens. Contrast sensitivity function for different spatial frequencies did not present any relevant or significant correlation with RPRE or HOA.

\section{Discussion}

The present study shows the efficacy of custom-designed RGP and soft $\mathrm{CL}$ to change the relative peripheral refractive error (RPRE) of myopic eyes. Overall, all the experimental conditions induced a change toward reducing peripheral hyperopia including a noncustomized aspheric RGP lens. However, the customized aspheric design of the experimental lenses were shown to be effective in moving the peripheral image shell forward, creating a myopization effect. This was achieved in part at the expense of an increase in peripheral astigmatism ( $\mathrm{J} 0$ component). There was some degree of asymmetry in the effect induced, particularly with the experimental RGP lens. This might be related with slight decentrations of the lens in primary gaze. Despite of the large diameter and the fitting process we cannot discard that small levels of decentration (within our criteria of $0.5 \mathrm{~mm}$ ) might affect our results and justify the asymmetry in the change in RPRE for the M component of refraction. This was particularly relevant for the ExpRGP. This finding is expected as the same degree of decentration would have a higher impact on the lenses with a more effective change of power from center to periphery. Good centration might be relevant to obtain a consistent peripheral myopic shift with the purpose of myopia regulation [20]. Previous studies showed only relative myopic defocus in the nasal visual field [13], and this could be related with systematic decentration of the contact lens. Instead, the soft contact lens used in this study showed a more symmetric effect when comparing the more peripheral eccentricities at $30^{\circ}$ in the Nasal and Temporal visual fields. Future lens designs must address also the asymmetry present between the nasal and temporal visual field in stable, but mainly in progressing myopes [25]. In this study we discard lens 
A

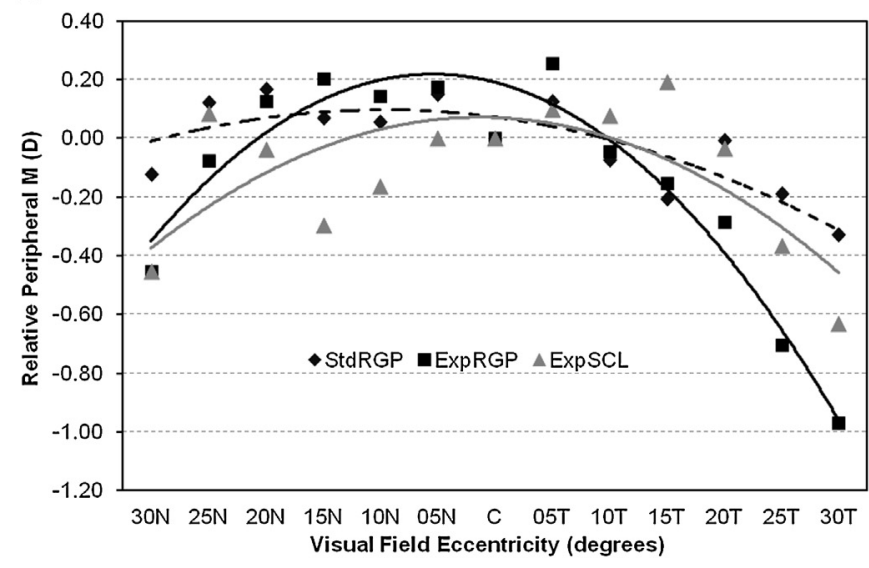

B

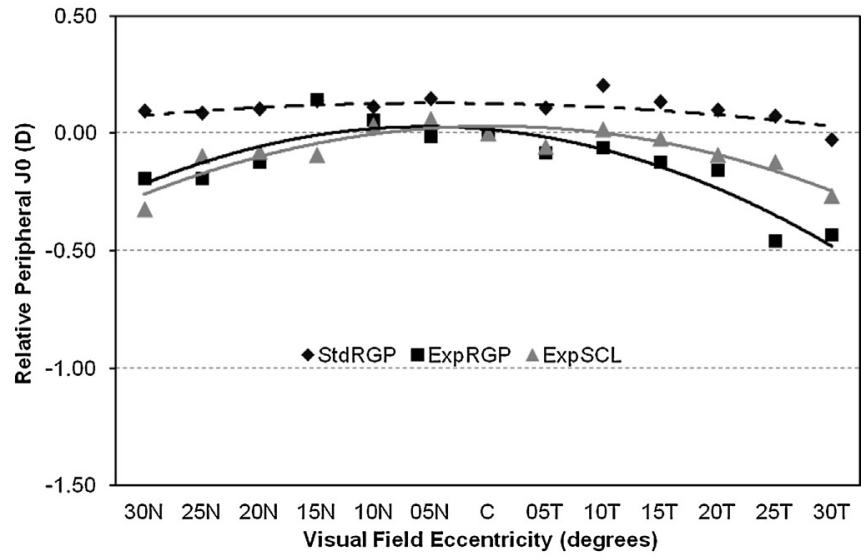

C

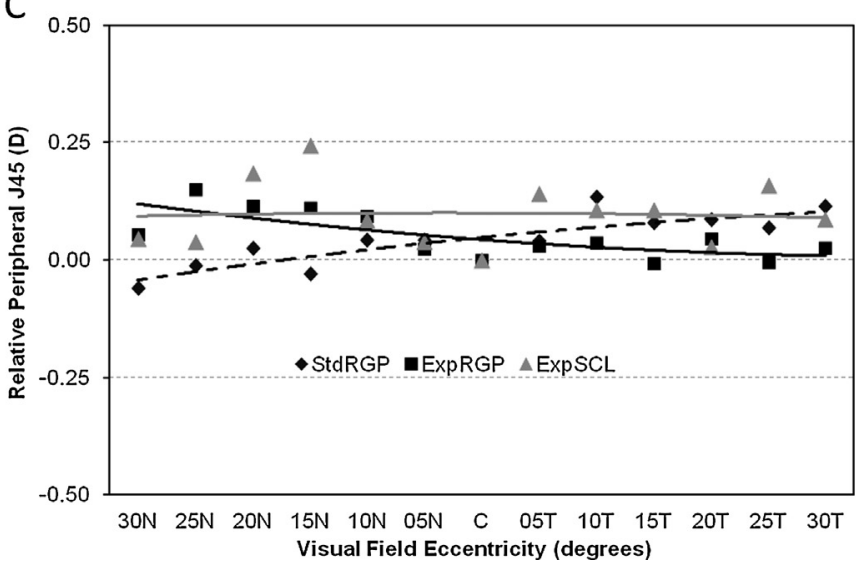

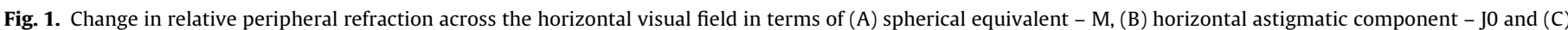

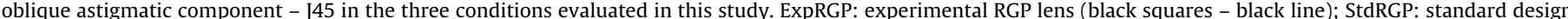

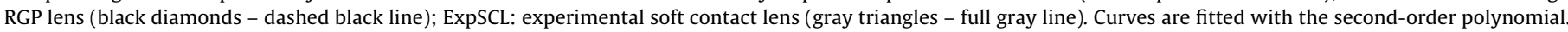
C: center; N: nasal; $\mathrm{T}$ : temporal.

decentration induced by eye turn as we modified the technique to measure the peripheral refraction by turning the head instead, and thus minimizing the interaction of the lids with the contact lenses on peripheral gaze. However, we cannot discard the contribution of slight decentrations (within $0.5 \mathrm{~mm}$ ) in primary gaze. This might have an effect on our results. Peripheral measures would be more myopic if the lens decenters in the direction of the retinal area being measured (i.e. nasal decentration when the nasal retina is being measured) and less myopic if the lens decenters in the opposite direction of the retinal area being measured (i.e. temporal decentration when the nasal retina is being measured). However, the fact that slightly hyperopic values are found to both sides of the central point, makes us realize that this is not the main cause. Optical quality measures might also reflect this slight decentrations by the
A

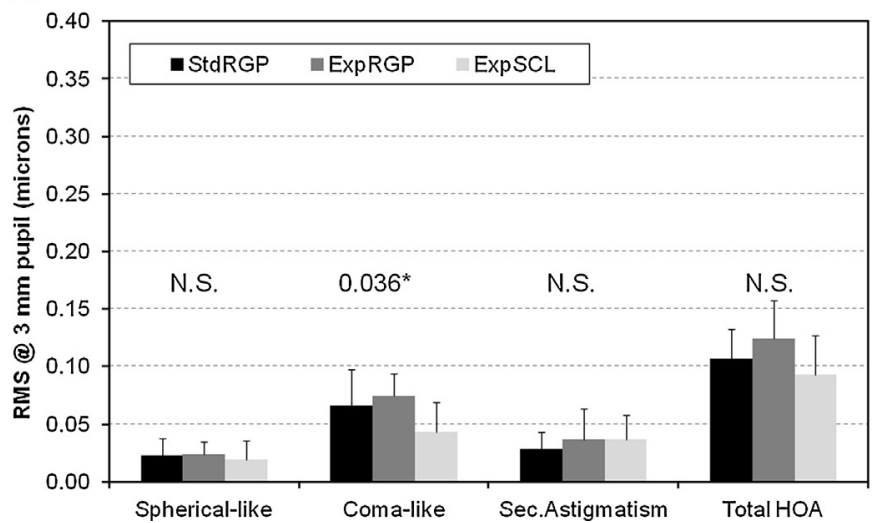

B

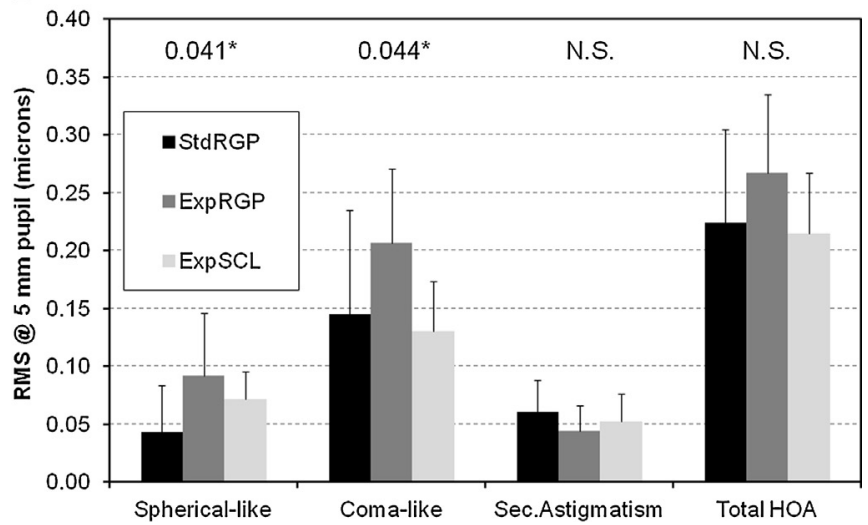

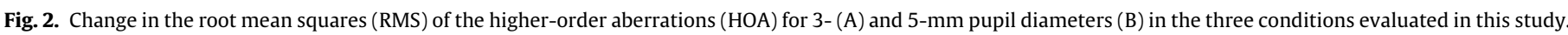
ExpRGP: experimental RGP lens; StdRGP: standard design RGP lens; ExpSCL: experimental soft contact lens. 
increased coma-like HOA observed for $3 \mathrm{~mm}$ and particularly for $5 \mathrm{~mm}$ pupil size. However, the changes induced in HOA were moderate and this is in agreement with the fact that all the experimental conditions maintained the CSF within the normal limits.

Although other lenses presenting a gradient of relatively positive power from the center to the periphery have demonstrated to be useful in decreasing myopia progression [13], the effectiveness of the lenses evaluated in this study for controlling myopic progression will have to be tested in a longitudinal clinical trial. Previous studies have demonstrated the effectiveness of orthokeratology in creating a peripheral myopic defocus $[9,10]$. The values of myopic change in RPRE reported for orthokeratology [10] are stronger than those obtained in the present study with the ExpRGP lens. However, orthokeratology has a limitation in lower myopes as the amount of RPRE is self-limited by the central refractive error being corrected [10]. This might be a reason for poorer myopic retention in lower myopes as observed in a previous clinical study [6]. Custom-designed lenses for daytime wear have the potential advantage of carrying any desired peripheral myopization effect irrespective of the central refractive error. This is the case for multifocal soft contact lenses of different add powers [15,16]. When compared with other peripheral gradient designs [13], and multifocal center-distance designs [16,26], the amount of change in RPRE is similar for the ExpSoft. There is not any other peripheral gradient RGP lens design in the market to compare our results but our results indicate that the potential of RPRE change with RGP designs is much efficient compared to the equivalent designs made in soft materials.

Beyond the value of custom-designed RGP and SCL, the present study also supports the use of non-custom designed RGP lenses to create a modest but statistically significant degree of change in RPRE. Although conventional design RGP lenses did not provided evidence of myopic retention against soft $\mathrm{CL}$ wearers in a clinical trial [27], the present results suggests that might be some benefit of RGP lens wear against conventional spectacle correction. Spectacle lenses were found to exacerbate the hyperopic defocus in myopic subjects [28-30]. Instead, Shen et al. [31] have recently measured the peripheral defocus in myopic subjects wearing conventional designed RGP and soft CL. They found that RGP lenses reduced significantly the relative peripheral hyperopia and they were more effective than soft CL on this regard. In the present study we did not use a standard soft CL to compare with the experimental soft CL. However, according to previous reports we would assume that single vision SCL do not significantly change the pattern of relative peripheral refractive errors [15,32]. Accordingly, the potential benefit assumed for StdRGP lenses might not apply to a single vision (standard) SCL based on our previous reports of no significant change in RPRE with a center-distance multifocal contact lens with 1 diopter of add [15]. This might be different for higher myopes, as Kwok et al. [33] have recently found that correcting high myopia with soft contact lenses might result in a significantly more myopic defocus in the retinal periphery.

Overall, the myopization effect created in CL which aimed for interfering with myopic progression, has a minimal impact in the optical quality of the visual system for a $5 \mathrm{~mm}$ pupil diameter with non-clinically significant changes in contrast sensitivity function (all conditions showed CSF within normal limits). The absence of correlations between CSF and RPRE induced by the experimental RGP or soft contact lens targeted in this experiment can be considered an expected result as CSF remained within normal limits and non-significantly different from baseline (naked eye) for the two experimental lenses evaluated. Despite this good outcome, the potential effect of the optical design of the lenses on the optical quality of the eye might worsen for pupil diameters larger than a $5-\mathrm{mm}$ pupil, which might be present in a significant number of young subjects even under mesopic and scotopic conditions.
Kollbaum et al. [34] have recently observed that low contrast acuity is not affected when tested at high illumination conditions but could be slightly affected under low illumination conditions with the Proclear MF contact lens. However, we cannot extrapolate their results to the present lens designs as they are different lenses and Proclear MF is not designed by purpose for myopia control, although used off-label with this purpose [35].

The practical implications of the present work are related with the platform of choice to create myopia regulation optical treatments. This might consider the interaction between centration, comfort and stability of the intended design to achieve a desired effect. Moreover, the level of complexity can be increased if we consider the asymmetries of the retinal profile across the horizontal visual field, and also between the horizontal [25] and less explored vertical orientations in myopic eyes [2]. Nevertheless, centration would be always of paramount importance and future devices must incorporate the efficacy in changing the peripheral refractive pattern achieved with experimental RGP lenses with the centration and comfort provided by soft contact lenses. From the quality of vision point of view, no particular concerns are raised by the use of these lenses. We have measured the optical quality of the lens + eye system and despite the expected increase in higher order aberrations, these aberrations did not had a clinically relevant impact in the visual quality measured with the contrast sensitivity test. This might be explained because of the pupil size of the subjects was not large enough to allow the peripheral gradient power to affect foveal vision. This is a relevant observation considering that this is the first study measuring the contrast sensitivity function with devices intended to be used for myopia regulation.

In conclusion, the study shows promising results for these custom-made lenses although ExpRGP might be preferred in terms of effectiveness for bringing the peripheral retinal image forward in order to interfere with myopic progression with minimal impact in terms of optical quality and visual performance.

\section{Conflict of interest}

Jaume Pauné has proprietary and financial interests in the manufacturing and distribution of the experimental lenses evaluated in this study. The remaining authors declare that they do not have any proprietary or financial interest in any of the materials mentioned in this article.

\section{Acknowledgements}

Paunevision (Spain) sponsored the present study. This work was also funded in part by FEDER (COMPETE Program) in the framework of projects PTDC/SAU-BEB/098391/2008, PTDC/SAUBEB/098392/2008 and Strategic Project PEST-C/FIS/UI607/2011 granted throught the Portuguese Foundation for Science and Technology (FCT).

\section{References}

[1] Huang J, Hung LF, Ramamirtham R, Blasdel TL, Humbird TL, Bockhorst KH, et al. Effects of form deprivation on peripheral refractions and ocular shape in infant rhesus monkeys (Macaca mulatta). Investig Ophthalmol Vis Sci 2009;50:4033-44.

[2] Atchison DA, Pritchard N, Schmid KL. Peripheral refraction along the horizontal and vertical visual fields in myopia. Vis Res 2006;46:1458.

[3] Ehsaei A, Mallen EA, Chisholm CM, Pacey IE. Cross-sectional sample of peripheral refraction in four meridians in myopes and emmetropes. Investig Ophthalmol Vis Sci 2011;52:7574-85.

[4] Chen X, Sankaridurg P, Donovan L, Lin Z, Li L, Martinez A, et al. Characteristics of peripheral refractive errors of myopic and non-myopic Chinese eyes. Vis Res 2010;50:31-5.

[5] Kang P, Gifford P, McNamara P, Wu J, Yeo S, Vong B, et al. Peripheral refraction in different ethnicities. Investig Ophthalmol Vis Sci 2010;51:6059-65. 
[6] Cho P, Cheung SW, Edwards M. The longitudinal orthokeratology research in children (LORIC) in Hong Kong: a pilot study on refractive changes and myopic control. Curr Eye Res 2005;30:71-80.

[7] Kakita T, Hiraoka T, Oshika T. Influence of overnight orthokeratology on axial elongation in childhood myopia. Investig Ophthalmol Vis Sci 2011;52: 2170-4.

[8] Walline JJ, Jones LA, Sinnott LT. Corneal reshaping and myopia progression. Br J Ophthalmol 2009;93:1181-5.

[9] Charman WN, Mountford J, Atchison DA, Markwell EL. Peripheral refraction in orthokeratology patients. Optom Vis Sci 2006;83:641-8.

[10] Queirós A, González-Méijome JM, Jorge J, Villa-Collar C, Gutiérrez AR. Peripheral refraction in myopic patients after orthokeratology. Optom Vis Sci 2010;87:323-9.

[11] Tabernero J, Vazquez D, Seidemann A, Uttenweiler D, Schaeffel F, Effects of myopic spectacle correction and radial refractive gradient spectacles on peripheral refraction. Vis Res 2009;49:2176-86.

[12] Sankaridurg P, Donovan L, Varnas S, Ho A, Chen X, Martinez A, et al. Spectacle lenses designed to reduce progression of myopia: 12-month results. Optom Vis Sci 2010;87:631-41.

[13] Sankaridurg P, Holden B, Smith 3rd E, Naduvilath T, Chen X, de la Jara PL, et al. Decrease in rate of myopia progression with a contact lens designed to reduce relative peripheral hyperopia: one-year results. Investig Ophthalmol Vis Sci 2011;52:9362-7

[14] Wei X, Thibos L. Designing contact lenses for a wide field of view via ocular wavefront tomography. I Optom 2010:3:125-33.

[15] Lopes-Ferreira D, Ribeiro C, Maia R, García-Porta N, Queirós A, Villa-Collar C, et al. Peripheral myopization using a dominant design multifocal contact lens. J Optom 2011;4:14-21.

[16] Lopes-Ferreira D, Ribeiro C, Neves H, Faria-Ribeiro M, Queirós A, Villa-Collar C, et al. Peripheral refraction with dominant design multifocal contact lenses in young myopes. J Optom 2013;6:85-94.

[17] Anstice NS, Phillips JR. Effect of dual-focus soft contact lens wear on axial myopia progression in children. Ophthalmology 2011;118:1152-61.

[18] Atchison DA. Optical models for human myopic eyes. Vis Res 2006;46:2236-50.

[19] Wolffsohn JS, Hunt OA, Basra AK. Simplified recording of soft contact lens fit. Contact Lens Anterior Eye 2009;32:37-42.

[20] Smith III EL. Optical treatment strategies to slow myopia progression: effects of the visual extent of the optical treatment zone. Exp Eye Res 2013;114:77-88.
[21] Queiros A, Gonzalez-Meijome J, Jorge J. Influence of fogring lenses and cycloplegia on open-field automatic refraction. Ophthalmic Physiol Opt 2008;28:387-92.

[22] Mallen EA, Wolffsohn JS, Gilmartin B, Tsujimura S. Clinical evaluation of the Shin-Nippon SRW-5000 autorefractor in adults. Ophthalmic Physiol Opt $2001 ; 21: 101-7$

[23] Queiros A, Jorge J, Gonzalez-Meijome JM. Influence of fogging lenses and cycloplegia on peripheral refraction. J Optom 2009;2:83-9.

[24] Thibos LN, Wheeler W, Horner D. Power vectors: an application of Fourier anal ysis to the description and statistical analysis of refractive error. Optom Vis Sc 1997:74:367-75

[25] Faria-Ribeiro M, Queirós A, Lopes-Ferreira D, Jorge J, González-Méijome JM. Peripheral refraction and retinal contour in stable and progressive myopia. Optom Vis Sci 2013;90:9-15

[26] Ticak A, Walline JJ. Peripheral optics with bifocal soft and corneal reshaping contact lenses. Optom Vis Sci 2013;90:3-8.

[27] Walline JJ, Jones LA, Mutti DO, Zadnik K. A randomized trial of the effects of rigid contact lenses on myopia progression. Arch Ophthalmol 2004;122:1760-6.

[28] Lin Z, Martinez A, Chen X, Li L, Sankaridurg P, Holden B, et al. Periphera defocus with single-vision spectacle lenses in myopic children. Optom Vis Sci 2010;87:4-9.

[29] Bakaraju RC, Ehrmann K, Ho A, Papas EB. Pantoscopic tilt in spectaclecorrected myopia and its effect on peripheral refraction. Ophthalmic Physiol Opt 2008;28:538-49.

[30] Bakaraju RC, Ehrmann K, Papas E, Ho A. Do peripheral refraction and aberrations profiles vary with the type of myopia? - an illustration using a ray-tracing approach. J Optom 2009;2:29-38.

[31] Shen J, Clark CA, Soni PS, Thibos LN. Peripheral refraction with and without contact lens correction. Optom Vis Sci 2010;87:642-55.

[32] Davies LN, Mallen EA. Influence of accommodation and refractive status on the peripheral refractive profile. Br J Ophthalmol 2009;93:1186-90.

[33] Kwok E, Patel B, Backhouse S, Phillips JR. Peripheral refraction in high myopia with spherical soft contact lenses. Optom Vis Sci 2012;89:263-70.

[34] Kollbaum PS, Jansen ME, Tan J, Meyer DM, Rickert ME. Vision performance with a contact lens designed to slow myopia progression. Optom Vis Sci 2013:90:205-14

[35] Walline JJ, Greiner KL, McVey ME, Jones-Jordan LA. Multifocal contact lens myopia control. Optom Vis Sci 2013;90:1207-14 